\title{
Necessary and Sufficient Conditions for the Existence of Positive Solution for Singular Boundary Value Problems on Time Scales
}

\author{
Meiqiang Feng, ${ }^{1}$ Xuemei Zhang, ${ }^{2,3}$ Xianggui Li, ${ }^{1}$ and Weigao $\mathrm{Ge}^{3}$ \\ ${ }^{1}$ School of Science, Beijing Information Science \& Technology University, Beijing 100192, China \\ ${ }^{2}$ Department of Mathematics and Physics, North China Electric Power University, Beijing 102206, China \\ ${ }^{3}$ Department of Applied Mathematics, Beijing Institute of Technology, Beijing 100081, China
}

Correspondence should be addressed to Xuemei Zhang, zxm74@sina.com

Received 27 March 2009; Revised 3 July 2009; Accepted 15 September 2009

Recommended by Alberto Cabada

By constructing available upper and lower solutions and combining the Schauder's fixed point theorem with maximum principle, this paper establishes sufficient and necessary conditions to guarantee the existence of $C_{l d}[0,1]_{\mathbb{T}}$ as well as $C_{l d}^{\Delta}[0,1]_{\mathbb{T}}$ positive solutions for a class of singular boundary value problems on time scales. The results significantly extend and improve many known results for both the continuous case and more general time scales. We illustrate our results by one example.

Copyright (c) 2009 Meiqiang Feng et al. This is an open access article distributed under the Creative Commons Attribution License, which permits unrestricted use, distribution, and reproduction in any medium, provided the original work is properly cited.

\section{Introduction}

Recently, there have been many papers working on the existence of positive solutions to boundary value problems for differential equations on time scales; see, for example, [122]. This has been mainly due to its unification of the theory of differential and difference equations. An introduction to this unification is given in $[10,14,23,24]$. Now, this study is still a new area of fairly theoretical exploration in mathematics. However, it has led to several important applications, for example, in the study of insect population models, neural networks, heat transfer, and epidemic models; see, for example, $[9,10]$.

Motivated by works mentioned previously, we intend in this paper to establish sufficient and necessary conditions to guarantee the existence of positive solutions for the singular dynamic equation on time scales:

$$
x^{\Delta \nabla}+f(t, x)=0, \quad t \in(0,1)_{\mathbb{T}},
$$


subject to one of the following boundary conditions:

$$
x(0)=x(1)=0,
$$

or

$$
x(0)=x^{\Delta}(1)=0,
$$

where $\mathbb{T}$ is a time scale, $(0,1)_{\mathbb{T}}=(0,1) \cap \mathbb{T}$, where 0 is right dense and 1 is left dense. and $(H) f:(0,1)_{\mathbb{T}} \times[0,+\infty) \rightarrow[0,+\infty)$ is continuous. Suppose further that $f(t, x)$ is nonincreasing with respect to $x$, and there exists a function $g(k):[0,1] \rightarrow[1, \infty)$ such that

$$
f(t, k x) \leq g(k) f(t, x), \quad \forall(t, x) \in(0,1)_{\mathbb{T}} \times[0,+\infty)
$$

A necessary and sufficient condition for the existence of $C_{l d}[0,1]_{\mathbb{T}}$ as well as $C_{l d}^{\Delta}[0,1]_{\mathbb{T}}$ positive solutions is given by constructing upper and lower solutions and with the maximum principle. The nonlinearity $f(t, x)$ may be singular at $t=0$ and /or $t=1$. By singularity we mean that the functions $f$ in (1.1) is allowed to be unbounded at the points $t=0$ and / or $t=1$. A function $x(t) \in C_{l d}[0,1]_{\mathbb{T}} \cap C_{l d}^{\Delta \nabla}(0,1)_{\mathbb{T}}$ is called a $C_{l d}[0,1]_{\mathbb{T}}$ (positive) solution of (1.1) if it satisfies (1.1) $\left(x(t)>0\right.$, for $\left.t \in(0,1)_{\mathbb{T}}\right)$; if even $x^{\Delta}\left(0^{+}\right), x^{\Delta}\left(1^{-}\right)$exist, we call it is a $C_{l d}^{\Delta}[0,1]_{\mathbb{T}}$ solution.

To the best of our knowledge, there is very few literature giving sufficient and necessary conditions to guarantee the existence of positive solutions for singular boundary value problem on time scales. So it is interesting and important to discuss these problems. Many difficulties occur when we deal with them. For example, basic tools from calculus such as Fermat's theorem, Rolle's theorem, and the intermediate value theorem may not necessarily hold. So we need to introduce some new tools and methods to investigate the existence of positive solutions for problem (1.1) with one of the above boundary conditions.

The time scale related notations adopted in this paper can be found, if not explained specifically, in almost all literature related to time scales. The readers who are unfamiliar with this area can consult, for example, $[6,11-13,25,26]$ for details.

The organization of this paper is as follows. In Section 2, we provide some necessary background. In Section 3, the main results of problem (1.1)-(1.2) will be stated and proved. In Section 4, the main results of problem (1.1)-(1.3) will be investigated. Finally, in Section 5, one example is also included to illustrate the main results.

\section{Preliminaries}

In this section we will introduce several definitions on time scales and give some lemmas which are useful in proving our main results.

Definition 2.1. A time scale $\mathbb{T}$ is a nonempty closed subset of $R$. 
Definition 2.2. Define the forward (backward) jump operator $\sigma(t)$ at $t$ for $t<\sup \mathbb{T}(\rho(t)$ at $t$ for $t>\inf \mathbb{T}$ ) by

$$
\sigma(t)=\inf \{\tau>t: \tau \in \mathbb{T}\}(\rho(t)=\sup \{\tau<t: \tau \in \mathbb{T}\})
$$

for all $t \in \mathbb{T}$. We assume throughout that $\mathbb{T}$ has the topology that it inherits from the standard topology on $R$ and say $t$ is right scattered, left scattered, right dense and left dense if $\sigma(t)>$ $t, \rho(t)<t, \sigma(t)=t$, and $\rho(t)=t$, respectively. Finally, we introduce the sets $\mathbb{T}^{k}$ and $\mathbb{T}_{k}$ which are derived from the time scale $\mathbb{T}$ as follows. If $\mathbb{T}$ has a left-scattered maximum $t_{1}$, then $\mathbb{T}^{k}=$ $\mathbb{T}-t_{1}$, otherwise $\mathbb{T}^{k}=\mathbb{T}$. If $\mathbb{T}$ has a right-scattered minimum $t_{2}$, then $\mathbb{T}_{k}=\mathbb{T}-t_{2}$, otherwise $\mathbb{T}_{k}=\mathbb{T}$.

Definition 2.3. Fix $t \in \mathbb{T}$ and let $y: \mathbb{T} \rightarrow R$. Define $y^{\Delta}(t)$ to be the number (if it exists) with the property that given $\varepsilon>0$ there is a neighborhood $U$ of $t$ with

$$
\left|[y(\sigma(t))-y(s)]-y^{\Delta}(t)[\sigma(t)-s]\right|<\varepsilon|\sigma(t)-s|
$$

for all $s \in U$, where $y^{\Delta}$ denotes the (delta) derivative of $y$ with respect to the first variable, then

$$
g(t):=\int_{a}^{t} \omega(t, \tau) \Delta \tau
$$

implies

$$
g^{\Delta}(t)=\int_{a}^{t} \omega^{\Delta}(t, \tau) \Delta \tau+\omega(\sigma(t), \tau)
$$

Definition 2.4. Fix $t \in \mathbb{T}$ and let $y: \mathbb{T} \rightarrow R$. Define $y^{\nabla}(t)$ to be the number (if it exists) with the property that given $\varepsilon>0$ there is a neighborhood $U$ of $t$ with

$$
\left|[y(\rho(t))-y(s)]-y^{\nabla}(t)[\rho(t)-s]\right|<\varepsilon|\rho(t)-s|
$$

for all $s \in U$. Call $y^{\nabla}(t)$ the (nabla) derivative of $y(t)$ at the point $t$.

If $\mathbb{T}=\mathbb{R}$ then $f^{\Delta}(t)=f^{\nabla}(t)=f^{\prime}(t)$. If $\mathbb{T}=\mathbb{Z}$ then $f^{\Delta}(t)=f(t+1)-f(t)$ is the forward difference operator while $f^{\nabla}(t)=f(t)-f(t-1)$ is the backward difference operator.

Definition 2.5. A function $f: \mathbb{T} \rightarrow R$ is called rd-continuous provided that it is continuous at all right-dense points of $\mathbb{T}$ and its left-sided limit exists (finite) at left-dense points of $\mathbb{T}$. We let $C_{\text {rd }}^{0}(\mathbb{T})$ denote the set of rd-continuous functions $f: \mathbb{T} \rightarrow R$.

Definition 2.6. A function $f: \mathbb{T} \rightarrow R$ is called $l d$-continuous provided that it is continuous at all left-dense points of $\mathbb{T}$ and its right-sided limit exists (finite) at right-dense points of $\mathbb{T}$. We let $C_{l d}(\mathbb{T})$ denote the set of $l d$-continuous functions $f: \mathbb{T} \rightarrow R$. 
Definition 2.7. A function $F: \mathbb{T}^{k} \rightarrow R$ is called a delta-antiderivative of $f: \mathbb{T}^{k} \rightarrow R$ provided that $F^{\Delta}(t)=f(t)$ holds for all $t \in \mathbb{T}^{k}$. In this case we define the delta integral of $f$ by

$$
\int_{a}^{t} f(s) \Delta s=F(t)-F(a)
$$

for all $a, t \in \mathbb{T}$.

Definition 2.8. A function $\Phi: \mathbb{T}_{k} \rightarrow R$ is called a nabla-antiderivative of $f: \mathbb{T}_{k} \rightarrow R$ provided that $\Phi^{\nabla}(t)=f(t)$ holds for all $t \in \mathbb{T}_{k}$. In this case we define the delta integral of $f$ by

$$
\int_{a}^{t} f(s) \nabla s=\Phi(t)-\Phi(a)
$$

for all $a, t \in \mathbb{T}$.

Throughout this paper, we assume that $\mathbb{T}$ is a closed subset of $R$ with $0,1 \in \mathbb{T}$.

Let $E=C_{l d}[0,1]_{\mathbb{T}}$, equipped with the norm

$$
\|x\|:=\sup _{t \in[0,1]_{\mathbb{T}}}|x(t)|
$$

It is clear that $E$ is a real Banach space with the norm.

Lemma 2.9 (Maximum Principle). Let $a, b \in[0,1]_{\mathbb{T}}$ and $a<b$. If $x \in C_{l d}[0,1]_{\mathbb{T}} \cap C_{l d}^{\Delta \nabla}(0,1)_{\mathbb{T}}$, $x(a) \geq 0, x(b) \geq 0$, and $x^{\Delta \nabla}(t) \leq 0, t \in(a, b)_{\mathbb{T}}$. Then $x(t) \geq 0, t \in[a, b]_{\mathbb{T}}$.

\section{Existence of Positive Solution to (1.1)-(1.2)}

In this section, by constructing upper and lower solutions and with the maximum principle Lemma 2.9, we impose the growth conditions on $f$ which allow us to establish necessary and sufficient condition for the existence of (1.1)-(1.2).

We know that

$$
G(t, s)= \begin{cases}s(1-t), & \text { if } 0 \leq s \leq t \leq 1 \\ t(1-s), & \text { if } 0 \leq t \leq s \leq 1\end{cases}
$$

is the Green's function of corresponding homogeneous BVP of (1.1)-(1.2).

We can prove that $G(t, s)$ has the following properties.

Proposition 3.1. For $(t, s) \in[0,1]_{\mathbb{T}} \times[0,1]_{\mathbb{T}}$, one has

$$
\begin{gathered}
G(t, s) \geq 0 \\
e(t) e(s) \leq G(t, s) \leq G(t, t)=t(1-t)=e(t) .
\end{gathered}
$$


To obtain positive solutions of problem (1.1)-(1.2), the following results of Lemma 3.2 are fundamental.

Lemma 3.2. Assume that $(H)$ holds. If $\int_{0}^{t_{0}} \nabla s \int_{0}^{s} f(s, \bar{u}) \Delta t$ and $\int_{0}^{t_{0}} \Delta t \int_{t}^{t_{0}} f(s, \bar{u}) \nabla s$ exist and are finite, then one has

$$
\int_{0}^{t_{0}} \nabla s \int_{0}^{s} f(s, \bar{u}) \Delta t=\int_{0}^{t_{0}} \Delta t \int_{t}^{t_{0}} f(s, \bar{u}) \nabla s
$$

Proof. Without loss of generality, we suppose that there is only one right-scattered point $t_{1} \in$ $[0,1]_{\mathbb{T}}$. Then we have

$$
\begin{aligned}
\int_{0}^{t_{0}} \nabla s \int_{0}^{s} f(s, \bar{u}) \Delta t= & \int_{0}^{t_{1}} \nabla s \int_{0}^{s} f(s, \bar{u}) \Delta t+\int_{\sigma\left(t_{1}\right)}^{t_{0}} \nabla s \int_{0}^{s} f(s, \bar{u}) \Delta t+\int_{t_{1}}^{\sigma\left(t_{1}\right)} \nabla s \int_{0}^{s} f(s, \bar{u}) \Delta t \\
= & \int_{0}^{t_{1}} \Delta t \int_{t}^{t_{1}} f(s, \bar{u}) \nabla s+\int_{0}^{\sigma\left(t_{1}\right)} \Delta t \int_{\sigma\left(t_{1}\right)}^{t_{0}} f(s, \bar{u}) \nabla s \\
& +\int_{\sigma\left(t_{1}\right)}^{t_{0}} \Delta t \int_{t}^{t_{0}} f(s, \bar{u}) \nabla s+\mu\left(t_{1}\right) f\left(\sigma\left(t_{1}\right), \bar{u}\right) \sigma\left(t_{1}\right) \\
= & \int_{0}^{t_{1}} \Delta t \int_{t}^{t_{1}} f(s, \bar{u}) \nabla s+\int_{\sigma\left(t_{1}\right)}^{t_{0}} \Delta t \int_{t}^{t_{0}} f(s, \bar{u}) \nabla s+\sigma\left(t_{1}\right) \int_{\sigma\left(t_{1}\right)}^{t_{0}} f(s, \bar{u}) \nabla s \\
& +\mu\left(t_{1}\right) f\left(\sigma\left(t_{1}\right), \bar{u}\right) \sigma\left(t_{1}\right), \\
\int_{0}^{t_{0}} \Delta t \int_{0}^{s} f(s, \bar{u}) \nabla s= & \int_{0}^{t_{1}} \Delta t \int_{t}^{t_{0}} f(s, \bar{u}) \nabla s+\int_{t_{1}}^{\sigma\left(t_{1}\right)} \Delta t \int_{t}^{t_{0}} f(s, \bar{u}) \nabla s+\int_{\sigma\left(t_{1}\right)}^{t_{0}} \Delta t \int_{t}^{t_{0}} f(s, \bar{u}) \nabla s \\
= & \int_{0}^{t_{1}} \Delta t \int_{t}^{t_{1}} f(s, \bar{u}) \nabla s+\int_{0}^{t_{1}} \Delta t \int_{t_{1}}^{t_{0}} f(s, \bar{u}) \nabla s+\mu\left(t_{1}\right) \int_{t_{1}}^{t_{0}} f(s, \bar{u}) \nabla s \\
& +\int_{\sigma\left(t_{1}\right)}^{t_{0}} \Delta t \int_{t}^{t_{0}} f(s, \bar{u}) \nabla s \\
= & \int_{0}^{t_{1}} \Delta t \int_{t}^{t_{1}} f(s, \bar{u}) \nabla s+\left(t_{1}+\mu\left(t_{1}\right)\right) \int_{t_{1}}^{t_{0}} f(s, \bar{u}) \nabla s+\int_{\sigma\left(t_{1}\right)}^{t_{0}} \Delta t \int_{t}^{t_{0}} f(s, \bar{u}) \nabla s \\
& +\mu\left(t_{1}\right) f\left(\sigma\left(t_{1}\right), \bar{u}\right) \sigma\left(t_{1}\right), \quad \sigma\left(t_{1}\right)\left[\int_{t_{1}}^{t_{1}} \Delta t \int_{t}^{t_{1}} f(s, \bar{u}) \nabla s+\int_{\sigma\left(t_{1}\right)}^{t_{0}} \Delta t \int_{t}^{t_{0}} f(s, \bar{u}) \nabla s\right. \\
= & \int_{0}^{t_{1}} \Delta t \int_{t}^{t_{1}} f(s, \bar{u}) \nabla s+\int_{\sigma\left(t_{1}\right)}^{t_{0}} \Delta t \int_{t}^{t_{0}} f(s, \bar{u}) \nabla s+\sigma\left(t_{1}\right) \int_{\sigma\left(t_{1}\right)}^{t_{0}} f(s, \bar{u}) \nabla s \\
& \left.\int_{0}^{t_{0}} f(s, \bar{u}) \nabla s\right] \\
& \\
&
\end{aligned}
$$


that is,

$$
\int_{0}^{t_{0}} \Delta t \int_{0}^{s} f(s, \bar{u}) \nabla s=\int_{0}^{t_{0}} \Delta t \int_{0}^{s} f(s, \bar{u}) \nabla s
$$

Similarly, we can prove

$$
\int_{\sigma\left(t_{0}\right)}^{1} \nabla s \int_{s}^{1} f(s, \bar{u}) \Delta t=\int_{\sigma\left(t_{0}\right)}^{1} \Delta t \int_{\sigma\left(t_{0}\right)}^{t} f(s, \bar{u}) \nabla s .
$$

The proof is complete.

Theorem 3.3. Suppose that $(H)$ holds. Then problem (1.1)-(1.2) has a $C_{l d}[0,1]_{\mathbb{T}}$ positive solution if and only if the following integral condition holds:

$$
0<\int_{0}^{1} e(s) f(s, 1) \nabla s<+\infty .
$$

Proof. (1) Necessity

By $(H)$, there exists $g(k):[0,1] \rightarrow[1, \infty)$ such that $f(t, k x) \leq g(k) f(t, x)$. Without loss of generality, we assume that $g(k)$ is nonincreasing on $[0,1]$ with $g(1) \geq 1$.

Suppose that $u$ is a positive solution of problem (1.1)-(1.2), then

$$
u^{\Delta \nabla}(t)=-f(t, u(t)) \leq 0
$$

which implies that $u$ is concave on $[0,1]_{\mathbb{T}}$. Combining this with the boundary conditions, we have $u^{\Delta}(0)>0, u^{\Delta}(1)<0$. Therefore $u^{\Delta}(0) u^{\Delta}(1)<0$. So by [10, Theorem 1.115], there exists $t_{0} \in(0,1)_{\mathbb{T}}$ satisfying $u^{\Delta}\left(t_{0}\right)=0$ or $u^{\Delta}\left(t_{0}\right) u^{\Delta}\left(\sigma\left(t_{0}\right)\right) \leq 0$. And $u^{\Delta}(t)>0$ for $t \in\left(0, t_{0}\right), u^{\Delta}(t)<0$, for $t \in\left(\sigma\left(t_{0}\right), 1\right)$. Denote $\bar{u}=\max \left\{u\left(t_{0}\right), u\left(\sigma\left(t_{0}\right)\right)\right\}$, then $\bar{u}=\max _{t \in[0,1]_{\mathbb{T}}} u(t)$.

First we prove $0<\int_{0}^{1} e(s) f(s, 1) \nabla s$.

By $(H)$, for any fixed $u, v>0$, we have

$$
f(t, u)=f\left(t, \frac{u}{v} v\right) \leq g\left(\frac{u}{v}\right) f(t, v), \quad u \leq v
$$

It follows that

$$
f(t, u) \leq g\left(\frac{2 u}{u+v+|u-v|}\right) f(t, v) \quad \forall u, v \in R^{+}=[0,+\infty)
$$


If $f(t, 1) \equiv 0$, then we have by (3.10)

$$
0 \leq f(t, u) \leq g\left(\frac{2 u}{u+1+|u-1|}\right) f(t, 1) \quad \forall t \in(0,1)_{\mathbb{T}}
$$

This means $f(t, u(t)) \equiv 0$, then $u(t) \equiv 0$, which is a contradiction with $u(t)$ being positive solution. Thus $f(t, 1) \not \equiv 0$, then $0<\int_{0}^{1} e(s) f(s, 1) \nabla s$.

Second, we prove $\int_{0}^{1} e(s) f(s, 1) \nabla s<+\infty$.

If $u^{\Delta}\left(t_{0}\right)=0$, then

$$
\begin{array}{ll}
\int_{t}^{t_{0}} f(s, u(s)) \nabla s=-\int_{t}^{t_{0}} u^{\Delta \nabla}(s) \nabla s=-u^{\Delta}\left(t_{0}\right)+u^{\Delta}(t)=u^{\Delta}(t) \quad \text { for } t \in\left(0, t_{0}\right) \\
\int_{t_{0}}^{t} f(s, u(s)) \nabla s=-\int_{t_{0}}^{t} u^{\Delta \nabla}(s) \nabla s=-u^{\Delta}(t)+u^{\Delta}\left(t_{0}\right)=-u^{\Delta}(t) \text { for } t \in\left(t_{0}, 1\right) .
\end{array}
$$

If $u^{\Delta}\left(t_{0}\right) u^{\Delta}\left(\sigma\left(t_{0}\right)\right)<0$, then $u^{\Delta}\left(t_{0}\right)>0, u^{\Delta}\left(\sigma\left(t_{0}\right)\right)<0$, and

$$
\begin{gathered}
\int_{t}^{t_{0}} f(s, u(s)) \nabla s=-\int_{t}^{t_{0}} u^{\Delta \nabla}(s) \nabla s=-u^{\Delta}\left(t_{0}\right)+u^{\Delta}(t) \leq u^{\Delta}(t) \quad \text { for } t \in\left(0, t_{0}\right) \\
\int_{\sigma\left(t_{0}\right)}^{t} f(s, u(s)) \nabla s=-\int_{\sigma\left(t_{0}\right)}^{t} u^{\Delta \nabla}(s) \nabla s=-u^{\Delta}(t)+u^{\Delta}\left(\sigma\left(t_{0}\right)\right) \leq-u^{\Delta}(t) \quad \text { for } t \in\left(\sigma\left(t_{0}\right), 1\right) .
\end{gathered}
$$

It follows that

$$
\begin{gathered}
\int_{t}^{t_{0}} f(s, \bar{u}) \nabla s \leq \int_{t}^{t_{0}} f(s, u(s)) \nabla s \leq u^{\Delta}(t) \quad \text { for } t \in\left(0, t_{0}\right) \\
\int_{\sigma\left(t_{0}\right)}^{t} f(s, \bar{u}) \nabla s \leq \int_{\sigma\left(t_{0}\right)}^{t} f(s, u(s)) \nabla s \leq-u^{\Delta}(t) \quad \text { for } t \in\left(\sigma\left(t_{0}\right), 1\right) .
\end{gathered}
$$


By (3.14) we have

$$
\begin{aligned}
\int_{0}^{t_{0}} s f(s, \bar{u}) \nabla s & =\int_{0}^{t_{0}} \nabla s \int_{0}^{s} f(s, \bar{u}) \Delta t \\
& =\int_{0}^{t_{0}} \Delta t \int_{t}^{t_{0}} f(s, \bar{u}) \nabla s \\
& \leq \int_{0}^{t_{0}} u^{\Delta}(t) \Delta t \\
& =u\left(t_{0}\right)-u(0) \\
& =u\left(t_{0}\right)<+\infty, \\
\int_{\sigma\left(t_{0}\right)}^{1}(1-s) f(s, \bar{u}) \nabla s & =\int_{\sigma\left(t_{0}\right)}^{1} \nabla s \int_{s}^{1} f(s, \bar{u}) \Delta t \\
& =\int_{\sigma\left(t_{0}\right)}^{1} \Delta t \int_{\sigma\left(t_{0}\right)}^{t} f(s, \bar{u}) \nabla s \\
& \leq-\int_{\sigma\left(t_{0}\right)}^{1} u^{\Delta}(t) \Delta t \\
& =u\left(\sigma\left(t_{0}\right)\right)-u(1) \\
& =u\left(\sigma\left(t_{0}\right)\right)<+\infty .
\end{aligned}
$$

Combining this with (3.10) we obtain

$$
\begin{aligned}
\int_{0}^{t_{0}} s f(s, 1) \nabla s & \leq \int_{0}^{t_{0}} s g\left(\frac{2}{1+\bar{u}+|1-\bar{u}|}\right) f(s, \bar{u}) \nabla s \\
& =g\left(\frac{2}{1+\bar{u}+|1-\bar{u}|}\right) \int_{0}^{t_{0}} s f(s, \bar{u}) \nabla s<+\infty .
\end{aligned}
$$

Similarly

$$
\int_{\sigma\left(t_{0}\right)}^{1}(1-s) f(s, 1) \nabla s<+\infty .
$$

Then we can obtain

$$
0<\int_{0}^{1} e(s) f(s, 1) \nabla s<+\infty .
$$


(2) Sufficiency

Let

$$
a(t)=\int_{0}^{1} G(t, s) f(s, 1) \nabla s, b(t)=\int_{0}^{1} G(t, s) f(s, e(s)) \nabla s
$$

Then

$$
\begin{gathered}
e(t) \int_{0}^{1} e(s) f(s, 1) \nabla s \leq a(t) \leq b(t) \leq \int_{0}^{1} e(s) f(s, e(s)) \nabla s, \\
a^{\Delta \nabla}(t)=-f(t, 1), \quad b^{\Delta \nabla}(t)=-f(t, e(t)) .
\end{gathered}
$$

Let

$$
k_{1}=\int_{0}^{1} e(s) f(s, 1) \nabla s, \quad l=\min \left\{1, k_{1}^{-1}\right\}, \quad L=\max \left\{1, k_{1}^{-1}\right\}, \quad k_{2}=\int_{0}^{1} e(s) f(s, e(s)) \nabla s,
$$

then $l \leq 1, L \geq 1$.

Let $H(t)=l a(t), Q(t)=L b(t)$, then

$$
l a(t) \leq l \int_{0}^{1} e(s) f(s, 1) \nabla s \leq 1, \quad L k_{1} e(t) \leq L b(t) \leq L k_{2} \triangleq \rho .
$$

So, we have

$$
\begin{aligned}
H^{\Delta \nabla}(t)+f(t, H(t)) & =f(t, l a(t))-l f(t, 1) \\
& \geq f(t, 1)-l f(t, 1) \geq 0, \\
Q^{\Delta \nabla}(t)+f(t, Q(t)) & =f(t, L b(t))-L f(t, e(t)) \\
& \leq f\left(t, L k_{1} e(t)\right)-L f(t, e(t)) \\
& \leq f(t, e(t))-L f(t, e(t)) \leq 0,
\end{aligned}
$$

and $H(0)=H(1)=Q(0)=Q(1)=0$. Hence $H(t), Q(t)$ are lower and upper solutions of problem (1.1)-(1.2), respectively. Obviously $H(t)>0$ for $t \in(0,1)_{\mathbb{T}}$.

Now we prove that problem (1.1)-(1.2) has a positive solution $x^{*} \in C_{l d}[0,1]_{\mathbb{T}}$ with $0<H(t) \leq x^{*} \leq Q(t)$.

Define a function

$$
F(t, x)=\left\{\begin{array}{l}
f(t, H(t)), \quad x<H(t) \\
f(t, x), \quad H(t) \leq x \leq Q(t) \\
f(t, Q(t)), \quad x>Q(t)
\end{array}\right.
$$


Then $F:(0,1)_{\mathbb{T}} \times R^{+} \rightarrow R^{+}$is continuous. Consider BVP

$$
\begin{gathered}
-x^{\Delta \nabla}(t)=F(t, x), \\
x(0)=x(1)=0 .
\end{gathered}
$$

Define mapping $A: E \rightarrow E$ by

$$
A x(t)=\int_{0}^{1} G(t, s) F(s, x(s)) \nabla s .
$$

Then problem (1.1)-(1.2) has a positive solution if and only if $A$ has a fixed point $x^{*} \in$ $C_{l d}[0,1]_{\mathbb{T}}$ with $0<H(t) \leq x^{*} \leq Q(t)$.

Obviously $A$ is continuous. Let $D=\left\{x \mid\|x\| \leq \rho^{*}, x \in E, \rho^{*} \in R^{+}\right\}$. By (3.7) and (3.16), for all $x \in D$, we have

$$
\begin{aligned}
\int_{0}^{1} G(t, s) F(s, x(s)) \nabla s & \leq \int_{0}^{1} G(t, s) f(s, H(s)) \nabla s \\
& \leq \int_{0}^{1} G(t, s) f(s, 0) \nabla s \\
& \leq g(0) \int_{0}^{1} G(t, s) f(t, 1) \nabla s \\
& \leq g(0) \int_{0}^{1} e(s) f(t, 1) \nabla s<+\infty .
\end{aligned}
$$

Then $A(D)$ is bounded. By the continuity of $G(t, s)$ we can easily found that $\{A u(t) \mid u(t) \in$ $D\}$ are equicontinuous. Thus $A$ is completely continuous. By Schauder fixed point theorem we found that $A$ has at least one fixed point $x^{*} \in D$.

We prove $0<H(t) \leq x^{*} \leq Q(t)$. If there exists $t_{*} \in(0,1)_{\mathbb{T}}$ such that

$$
x^{*}\left(t_{*}\right)>Q\left(t_{*}\right)
$$

Let $z(t)=Q(t)-x^{*}, c=\inf \left\{t_{1} \mid 0 \leq t_{1}<t_{*}, z(t)<0, \forall t \in\left(t_{1}, t_{*}\right]\right\}, d=\sup \left\{t_{2} \mid t_{*}<t_{2} \leq 1, z(t)<\right.$ $\left.0, \forall t \in\left(t_{*}, t_{2}\right]\right\}$ then $Q(t)<x^{*}$ for $t \in(c, d)_{\mathrm{T}}$. Thus $F\left(t, x^{*}\right)=f(t, Q(t)), t \in(c, d)_{\mathrm{T}}$. By (3.24) we know that $-z^{\Delta \nabla}(t)=Q^{\Delta \nabla}(t)-x^{\Delta \nabla}(t) \leq 0$. And $z(c)=Q(c)-x^{*}(c) \geq 0, z(d)=Q(d)-x^{*}(d) \geq 0$. By Lemma 2.9 we have $z(t) \geq 0, t \in[c, d]_{\mathbb{T}}$, which is a contradiction. Then $x^{*} \leq Q(t)$. Similarly we can prove $H(t) \leq x^{*}$. The proof is complete.

Theorem 3.4. Suppose that $(H)$ holds. Then problem (1.1)-(1.2) has a $C_{l d}^{\Delta}[0,1]_{\mathbb{T}}$ positive solution if and only if the following integral condition holds:

$$
0<\int_{0}^{1} f(s, e(s)) \nabla s<+\infty
$$


Proof. (1) Necessity

Let $u(t) \in C_{l d}^{\Delta}[0,1]_{\mathrm{T}}$ be a positive solution of problem (1.1)-(1.2). Then $u^{\Delta}(t)$ is decreasing on $[0,1]_{\mathrm{T}}$. Hence $u^{\Delta \nabla}(t)$ is integrable and

$$
\int_{0}^{1} f(t, u(t)) \nabla t=-\int_{0}^{1} u^{\Delta \nabla}(t) \nabla t<+\infty
$$

By simple computation and using [10, Theorem 1.119], we obtain $\lim _{t \rightarrow 0^{+}}(u(t) / e(t))>$ $0, \lim _{t \rightarrow 1^{-}}(u(t) / e(t))>0$. So there exist $M>1>m>0$ such that $m e(t) \leq u(t) \leq M e(t)$. By $(H)$ we obtain

$$
\begin{gathered}
g\left(M^{-1}\right)^{-1} f(t, e(t)) \leq f(t, M e(t)) \leq f(t, u(t)) \\
\int_{0}^{1} f(t, e(t)) \nabla t \leq g\left(M^{-1}\right) \int_{0}^{1} f(t, u(t)) \nabla t<\infty .
\end{gathered}
$$

By

$$
e(t) f(t, 1) \leq f(t, e(t)) \leq g(e(t)) f(t, 1),
$$

we have $0<\int_{0}^{1} e(t) f(t, 1) \nabla t \leq \int_{0}^{1} f(t, e(t)) \nabla t<\infty$.

(2) Sufficiency

Let $r(t)=\int_{0}^{1} G(t, s) f(s, e(s)) \nabla s$, then

$$
e(t) \int_{0}^{1} G(s, s) f(s, e(s)) \nabla s \leq r(t) \leq \int_{0}^{1} f(s, e(s)) \nabla s .
$$

Similar to Theorem 3.3, let $l^{\prime}=\min \left\{1, k_{2}^{-1}\right\}, L^{\prime}=\max \left\{1, k_{2}^{-1}\right\}, H(t)=l^{\prime} a(t), Q(t)=L^{\prime} r(t)$, there exists $\omega^{*}(t)$ satisfying $H(t) \leq \omega^{*}(t) \leq Q(t)$, and

$$
f\left(t, \omega^{*}(t)\right) \leq f(t, H(t)) \leq f\left(t, l^{\prime} k_{2} e(t)\right) \leq g\left(l^{\prime} k_{2}\right) f(t, e(t))
$$

then $\omega^{* \Delta \nabla}(t)$ is integral and $\omega^{* \Delta}(1-), \omega^{* \Delta}(0+)$ exist, hence $\omega^{*}(t)$ is a positive solution in $C_{l d}^{\Delta}[0,1]_{\mathrm{T}}$. The proof is complete. 


\section{Existence of Positive Solution to (1.1)-(1.3)}

Now we deal with problem (1.1)-(1.3). The method is just similar to what we have done in Section 3, so we omit the proof of main result of this section.

Let

$$
G_{1}(t, s)= \begin{cases}s & \text { if } 0 \leq s \leq t \leq 1 \\ t & \text { if } 0 \leq t \leq s \leq 1\end{cases}
$$

be the Green's function of corresponding homogeneous BVP of (1.1)-(1.3).

We can prove that $G_{1}(t, s)$ has the following properties.

Similar to (3.2), we have

$$
\begin{gathered}
G_{1}(t, s) \geq 0, \quad(t, s) \in[0,1]_{\mathbb{T}} \times[0,1]_{\mathbb{T}} \\
e_{1}(t) e_{1}(s) \leq G_{1}(t, s) \leq G_{1}(t, t)=t=e_{1}(t), \quad(t, s) \in[0,1]_{\mathbb{T}} \times[0,1]_{\mathbb{T}} .
\end{gathered}
$$

Theorem 4.1. Suppose that $(H)$ holds, then problem (1.1)-(1.3) has a $C_{l d}[0,1]_{\mathbb{T}}$ positive solution if and only if the following integral condition holds:

$$
0<\int_{0}^{1} e_{1}(s) f(s, 1) \nabla s<+\infty
$$

Theorem 4.2. Suppose that $(H)$ holds, then problem (1.1)-(1.3) has a $C_{l d}^{\Delta}[0,1]_{\mathbb{T}}$ positive solution if and only if the following integral condition holds:

$$
0<\int_{0}^{1} f\left(s, e_{1}(s)\right) \nabla s<+\infty
$$

\section{Example}

To illustrate how our main results can be used in practice we present an example.

Example 5.1. We have

$$
\begin{gathered}
-x^{\Delta \nabla}(t)=t^{-1 / 2} e^{-x}, \quad t \in(0,1)_{\mathbb{T}}, \\
x(0)=x(1)=0,
\end{gathered}
$$


where $f(t, x)=t^{-(1 / 2) e^{-x}}, \mathbb{T}=[0,1 / 2) \cup\{1 / 2,2 / 3,3 / 4, \ldots, n /(n+1), \ldots, 1\}$. Select $g(k)=$ $e(2-k), k \in[0,1]$, then we have $f(t, k x) \leq g(k) f(t, x), \forall(t, x) \in(0,1)_{\mathbb{T}} \times[0,+\infty)$. Moreover, we have

$$
\begin{aligned}
0<\int_{0}^{1} s(1-s) s^{-1 / 2} e^{-1} \nabla s & =e^{-1}\left[\frac{2}{3}\left(\frac{1}{2}\right)^{3 / 2}+\frac{2}{5}\left(\frac{1}{2}\right)^{5 / 2}+\sum_{n=1}^{\infty} \frac{1}{(n+1)^{(7 / 2)} n^{(1 / 2)}}\right] \\
& \leq e^{-1}\left[\sum_{n=1}^{\infty} \frac{1}{n^{4}}+\frac{2}{3}\left(\frac{1}{2}\right)^{3 / 2}+\frac{2}{5}\left(\frac{1}{2}\right)^{5 / 2}\right]<+\infty .
\end{aligned}
$$

By Theorem 3.3, problem (5.1) has a positive solution in $C_{l d}[0,1]_{\mathbb{T}}$.

Remark 5.2. Example 5.1 implies that there is a large number of functions that satisfy the conditions of Theorem 3.3. In addition, the conditions of Theorem 3.3 are also easy to check.

\section{Acknowledgments}

This work is sponsored by the National Natural Science Foundation of China (10671012, 10671023) and the Scientific Creative Platform Foundation of Beijing Municipal Commission of Education (PXM2008-014224-067420).

\section{References}

[1] D. R. Anderson, "Eigenvalue intervals for a two-point boundary value problem on a measure chain," Journal of Computational and Applied Mathematics, vol. 141, no. 1-2, pp. 57-64, 2002.

[2] R. I. Avery and D. R. Anderson, "Existence of three positive solutions to a second-order boundary value problem on a measure chain," Journal of Computational and Applied Mathematics, vol. 141, no. 1-2, pp. 65-73, 2002.

[3] F. M. Atici and G. Sh. Guseinov, "On Green's functions and positive solutions for boundary value problems on time scales," Journal of Computational and Applied Mathematics, vol. 141, no. 1-2, pp. 75-99, 2002.

[4] D. Anderson, R. Avery, and J. Henderson, "Existence of solutions for a one dimensional $p$-Laplacian on time-scales," Journal of Difference Equations and Applications, vol. 10, no. 10, pp. 889-896, 2004.

[5] M. Bohner and H. Luo, "Singular second-order multipoint dynamic boundary value problems with mixed derivatives," Advances in Difference Equations, vol. 2006, Article ID 54989, 15 pages, 2006.

[6] H. Luo and Q. Ma, "Positive solutions to a generalized second-order three-point boundary-value problem on time scales," Electronic Journal of Differential Equations, vol. 2005, no. 17, pp. 1-14, 2005.

[7] J. Henderson, "Multiple solutions for $2 m$ th order Sturm-Liouville boundary value problems on a measure chain," Journal of Difference Equations and Applications, vol. 6, no. 4, pp. 417-429, 2000.

[8] J. Henderson, A. Peterson, and C. C. Tisdell, "On the existence and uniqueness of solutions to boundary value problems on time scales," Advances in Difference Equations, vol. 2004, no. 2, pp. 93-109, 2004.

[9] R. P. Agarwal, M. Bohner, and W.-T. Li, Nonoscillation and Oscillation: Theory for Functional Differential Equations, vol. 267 of Monographs and Textbooks in Pure and Applied Mathematics, Marcel Dekker, New York, NY, USA, 2004.

[10] M. Bohner and A. Peterson, Dynamic Equations on Time Scales. An Introduction with Applications, Birkhäuser, Boston, Mass, USA, 2001.

[11] Z. He, "Double positive solutions of three-point boundary value problems for $p$-Laplacian dynamic equations on time scales," Journal of Computational and Applied Mathematics, vol. 182, no. 2, pp. 304-315, 2005. 
[12] Z. He and X. Jiang, "Triple positive solutions of boundary value problems for $p$-Laplacian dynamic equations on time scales," Journal of Mathematical Analysis and Applications, vol. 321, no. 2, pp. 911-920, 2006.

[13] Y. Tian and W. Ge, "Existence and uniqueness results for nonlinear first-order three-point boundary value problems on time scales," Nonlinear Analysis: Theory, Methods E Applications, vol. 69, no. 9, pp. 2833-2842, 2008.

[14] V. Lakshmikantham, S. Sivasundaram, and B. Kaymakcalan, Dynamic Systems on Measure Chains, vol. 370 of Mathematics and Its Applications, Kluwer Academic Publishers, Dordrecht, The Netherlands, 1996.

[15] L. Erbe, A. Peterson, and S. H. Saker, "Hille-Kneser-type criteria for second-order dynamic equations on time scales," Advances in Difference Equations, vol. 2006, Article ID 51401, 18 pages, 2006.

[16] R. A. Khan, J. J. Nieto, and V. Otero-Espinar, "Existence and approximation of solution of three-point boundary value problems on time scales," Journal of Difference Equations and Applications, vol. 14, no. 7, pp. 723-736, 2008.

[17] R. P. Agarwal, V. Otero-Espinar, K. Perera, and D. R. Vivero, "Multiple positive solutions in the sense of distributions of singular BVPs on time scales and an application to Emden-Fowler equations," Advances in Difference Equations, vol. 2008, Article ID 796851, 13 pages, 2008.

[18] D.-B. Wang, "Three positive solutions of three-point boundary value problems for $p$-Laplacian dynamic equations on time scales," Nonlinear Analysis: Theory, Methods $\mathcal{E}$ Applications, vol. 68, no. 8, pp. 2172-2180, 2008.

[19] J.-P. Sun, "A new existence theorem for right focal boundary value problems on a measure chain," Applied Mathematics Letters, vol. 18, no. 1, pp. 41-47, 2005.

[20] M. Feng, X. Zhang, and W. Ge, "Positive solutions for a class of boundary value problems on time scales," Computers \& Mathematics with Applications, vol. 54, no. 4, pp. 467-475, 2007.

[21] M.-Q. Feng, X.-G. Li, and W.-G. Ge, "Triple positive solutions of fourth-order four-point boundary value problems of $p$-Laplacian dynamic equations on time scales," Advances in Difference Equations, vol. 2008, Article ID 496078, 9 pages, 2008.

[22] M. Feng, H. Feng, X. Zhang, and W. Ge, "Triple positive solutions for a class of $m$-point dynamic equations on time scales with $p$-Laplacian," Mathematical and Computer Modelling, vol. 48, no. 7-8, pp. 1213-1226, 2008.

[23] M. Bohner and A. Peterson, Eds., Advances in Dynamic Equations on Time Scales, Birkhäuser, Boston, Mass, USA, 2003.

[24] S. Hilger, "Analysis on measure chains-a unified approach to continuous and discrete calculus," Results in Mathematics, vol. 18, no. 1-2, pp. 18-56, 1990.

[25] J. Henderson and C. C. Tisdell, "Topological transversality and boundary value problems on time scales," Journal of Mathematical Analysis and Applications, vol. 289, no. 1, pp. 110-125, 2004.

[26] Z. He, "Existence of two solutions of $m$-point boundary value problem for second order dynamic equations on time scales," Journal of Mathematical Analysis and Applications, vol. 296, no. 1, pp. 97-109, 2004. 